\title{
El derecho en la tierra, o la importancia del inicio de la frontera terrestre para definir fronteras marítimas. El caso entre el Perú y Chile
}

\author{
Fabián Novak Talavera* \\ Luis García-Corrochano Moyano*
}

\section{Introducción}

El presente artículo tiene como propósitos fundamentales, por un lado, establecer la importancia que tiene para la delimitación de las fronteras marítimas la definición del punto de la frontera terrestre desde el cual se dará inicio a dicha delimitación, y por otro lado, precisar cuál sería este punto terrestre en el caso de la frontera entre Perú y Chile que daría lugar al inicio de su frontera marítima.

Para tal efecto, será necesario analizar previamente las diversas posiciones que al respecto mantienen el Perú y Chile, explicando los fundamentos de hecho y de derecho que sostiene cada uno, así como las razones que determinan la validez de la posición peruana y llevan a descartar jurídicamente la chilena.

* (Lima, 1966), Profesor principal de Derecho Internacional Público de la Pontificia Universidad Católica del Perú. Ex Director e Investigador Principal del Instituto de Estudios Internacionales de la PUCP. Asociado del Instituto Hispano Luso Americano Filipino de Derecho Internacional (IHLADI). Miembro Asociado de la Sociedad Peruana de Derecho Internacional. Ex Asesor Jurídico del Ministerio de Relaciones Exteriores. Ex Viceministro de Políticas para la Defensa del Ministerio de Defensa.

** (Lima, 1968), Profesor de Derecho Internacional Público de la Academia Diplomática del Perú y de la Universidad Peruana de Ciencias Aplicadas (UPC). Coordinador de la Maestría en Relaciones Internacionales y Comercio, Instituto de Gobierno, Universidad San Martín de Porres. Miembro del Consejo Directivo del Instituto Hispano Luso Americano Filipino de Derecho Internacional (IHLADI). Miembro Asociado de la Sociedad Peruana de Derecho Internacional. Ex Asesor Jurídico del Ministerio de Relaciones Exteriores. Ex Viceministro de Justicia. 


\section{La determinación de la frontera terrestre en el Tratado y Proto- colo Complementario para resolver la cuestión de Tacna y Arica, firmados en Lima el 3 de junio de 1929, y la demarcación efectuada en 1930 por la Comisión Mixta de Límites entre el Perú y Chile}

Luego de una larga negociación cuyos pormenores se encuentran reseńados y documentados ${ }^{1}$, la frontera terrestre entre Perú y Chile quedó establecida por el Tratado y el Protocolo Complementario para resolver la cuestión de Tacna y Arica, firmados en Lima el 3 de junio de 1929, señalando el primero de ellos:

Artículo Segundo.- El Territorio de Tacna y Arica será dividido en dos partes, Tacna para el Perú y Arica para Chile. La línea divisoria entre dichas dos partes y; en consecuencia, la frontera entre los territorios del Perú y de Chile, partirá de un punto de la costa que se denominará "Concordia», distante diez kilómetros al norte del puente del río Lluta, para seguir para el oriente paralela a la vía de la sección chilena del ferrocarril de Arica a La Paz y distante diez kilómetros de ella, con las inflexiones necesarias para utilizar, en la demarcación, los accidentes geográficos cercanos que permitan dejar en territorio chileno las azufreras del Tacora y sus dependencias pasando luego por el centro de la Laguna Blanca, en forma que una de sus partes quede en el Perú y la otra en Chile. [...].

El Artículo Tercero del mismo instrumento jurídico estableció que la línea de frontera sería fijada y señalada en el territorio con hitos, por la Comisión Mixta de Límites entre el Perú y Chile, y que si se produjera algún desacuerdo en la comisión, sería resuelto con el voto dirimente de un tercer miembro designado por el Presidente de los Estados Unidos de América, cuyo fallo tendría el carácter de inapelable. El Artículo Cuarto estipuló la entrega de los territorios que eran devueltos al Perú, señalando que el acta correspondiente a dicha entrega «contendrá la relación detallada de la ubicación

1 Porras Barrenechea, Raúl y Alberto Wagner de Reyna. Historia de los límites del Perú. Lima: Fondo Editorial del Ministerio de Relaciones Exteriores del Perú, 1997. WaGner De ReYna, Alberto. Historia diplomática del Perú. Lima: Fondo Editorial del Ministerio de Relaciones Exteriores del Perú, 1997. UltoA, Alberto. Posición internacional del Perú. Lima: Fondo Editorial del Ministerio de Relaciones Exteriores del Perú, 1997. BÁkula, Juan Miguel. Perú: entre la realidad y la utopía. 180 años de política exterior. Tomo I. Lima: Fundación Academia Diplomática del Perú - Fondo de Cultura Económica, 2002. 
y características definitivas de los hitos fronterizos». La entrega de los territorios se verificó el 28 de agosto de 1929, y en el acta consta que:

Ambas Delegaciones convinieron que se firmaría, por separado, otra acta con la relación detallada de la ubicación y características definitivas de los hitos fronterizos conforme al artículo cuarto del Tratado de tres de junio de mil novecientos veintinueve, tan pronto como quedasen terminadas las operaciones de demarcación que debe realizar la respectiva Comisión Mixta.

La Comisión Mixta de Límites entre el Perú y Chile fue instalada el 2 de septiembre de 1929 por los delegados de ambos Estados, por el Perú Federico Basadre Grohmann y por Chile Enrique Brieba. Al momento de la demarcación surgieron discrepancias entre ambos delegados, en particular sobre el punto de inicio de la frontera en la costa, y sin necesidad de recurrir al Presidente de los Estados Unidos de América, de conformidad con el artículo tercero del Tratado para resolver la cuestión de Tacna y Arica, emitieron sus ministerios de Relaciones Exteriores sendas instrucciones de idéntico tenor a sus respectivos delegados para culminar la demarcación; la nota de instrucciones del Perú tiene fecha 24 de abril de 1930 y la nota de instrucciones de Chile está fechada el 28 de abril de 1930. El tenor de la nota chilena dirigida al delegado Brieba, concordante y coincidente con la nota peruana, es ilustrativo al respecto:

Para trazar la línea divisoria y colocar los hitos respectivos en los puntos que no alcanzaron a determinarse al suspender, en febrero último, los trabajos de la Comisión Mixta Demarcadora Chileno Peruana, se ha llegado a un acuerdo con el Gobierno de ese país, al cual deberá ajustarse esa Comisión en el trazado de la línea.

En esta virtud, en el desempeño de la misión que ha confiado a Ud. el Gobierno como Delegado de Chile en la Comisión Mixta de Límites con el Perú, se servirá Ud. dar cumplimiento a lo convenido y, de acuerdo con el Delegado del Perú, dispondrá que se proceda a la mayor brevedad a colocar los hitos divisorios en la línea que se determina, como sigue:

\section{HITO CONCORDIA.- Punto inicial en la costa de la línea fronteriza.}

Se medirán $10 \mathrm{Kms}$. desde el primer puente del Ferrocarril de Arica a La Paz sobre el río Lluta, en la dirección hacia el Norte en la Pampa de Escritos, y se trazará hacia el Poniente, un arco de diez kilómetros 
de radio, cuyo centro estará en el indicado puente y que vaya a interceptar la orilla del mar, de modo que, cualquier punto del arco, diste 10 kilómetros del referido puente del ferrocarril de Arica a La Paz sobre el río Lluta.

Este punto de intersección del arco trazado, con la orilla del mar, será el inicial de la línea divisoria entre Chile y el Perú.

Se colocará un hito en cualquier punto del arco, lo más próximo al mar posible, donde quede a cubierto de ser destruido por las aguas del océano.

Se aprecia de las instrucciones de ambos gobiernos dos aspectos que saltan a la vista. El primero es que ante la paralización de los trabajos demarcatorios por la existencia de desacuerdos, estos fueron definitivamente solucionados por entendimiento directo de los gobiernos de ambos Estados. El segundo es que la frontera entre el Perú y Chile se inicia en un punto de la costa, en la intersección del arco mencionado en las instrucciones aludidas con la orilla del mar. El Hito $\mathrm{N}^{\circ} 1$, que no es sino el primero de una sucesión de 80 hitos, fue colocado deliberadamente a cierta distancia tierra adentro a fin de que "quede a cubierto de ser destruido por las aguas del océano», tal como fuera expresamente dispuesto en dichas instrucciones.

El Acta Final de la Comisión Mixta de Límites entre el Perú y Chile, de 21 de julio de 1930, que lleva la firma de los mencionados delegados nacionales Basadre y Brieba, dejó en claro que: «se han colocado de mutuo acuerdo y en conformidad con las instrucciones recibidas por ambos delegados, todos los hitos necesarios para demarcar la línea de frontera entre el Perú y Chile, establecida por el tratado de Lima de fecha tres de junio de mil novecientos veintinueve.»; este documento perfecciona el Acta de entrega de Tacna de 28 de agosto de 1929, que de acuerdo a lo dispuesto por el Artículo Cuarto del Tratado, debía contener «la relación detallada de la ubicación y características definitivas de los hitos fronterizos». Asimismo, el Acta Final de la Comisión Mixta de Límites definió que «la línea de frontera demarcada parte del Océano Pacífico en un punto en la orilla del mar situado a diez kilómetros hacia el noroeste del primer puente sobre el río Lluta de la vía férrea de Arica a La Paz [...]». Luego, en la misma Acta se señala que "para fijar definitivamente la aludida línea de frontera entre Perú y Chile sobre el terreno, se han colocado o establecido hitos en la 
forma y posición que a continuación se indican [...] Hito $\mathrm{N}^{\circ} 1$, de concreto, Latitud Sur $18^{\circ} 21^{\prime} 03$ ", Longitud $70^{\circ} 22^{\prime}$ 56» ». Esta referencia se recoge en el Acta de 5 de agosto de 1930, suscrita por los ministros de Relaciones Exteriores de Perú y Chile.

Como se puede apreciar, el punto de la costa denominado Concordia y distante diez kilómetros al norte del puente del río Lluta, desde el cual había de partir la línea de frontera terrestre de conformidad con el artículo 3 del Tratado, fue ubicado por acuerdo directo entre los gobiernos en la intersección del arco con la orilla del mar, señalado en las idénticas instrucciones bajo comentario. Así, el texto de dichas instrucciones constituye una interpretación auténtica mediante la cual ambos gobiernos resolvieron las diferencias de interpretación que se produjeron en el seno de la Comisión Mixta de Límites y que no pudieron ser resueltas por los delegados de ambos países ante dicha Comisión Mixta.

De otro lado, de estos instrumentos también se desprende que el Hito $\mathrm{N}^{\circ} 1$ colocado a cierta distancia de la orilla, constituye la referencia visible más próxima a la orilla del mar, desde la cual, siguiendo el arco de 10 kilómetros hacia el noroeste del primer puente sobre el río Lluta de la vía férrea de Arica a La Paz, sobre el que se sitúan los demás hitos, se establece la línea de la frontera terrestre entre Perú y Chile.

Sobre este último punto es necesario precisar que el Hito $\mathrm{N}^{\circ} 1$ (Latitud $18^{\circ} 21^{\prime}$ 03» Sur), si bien constituye un hito de frontera, el más cercano al mar y, en consecuencia, el primero de una sucesión de 80 hitos, no constituye el punto inicial de dicha línea fronteriza. Esto queda meridianamente claro en las instrucciones por las cuales ambos Estados, de manera concordante y unívoca, interpretan el Tratado para resolver la cuestión de Tacna y Arica de 1929 estableciendo que la frontera terrestre se inicia en la orilla del mar, y que la demarcación será colocada «en cualquier punto del arco, lo más próximo posible a la orilla del mar», de lo que se aprecia que lo relevante para el establecimiento de la frontera es la determinación de su punto inicial, y no el lugar de colocación de los hitos o señales que den testimonio del curso de la frontera, en este caso, del arco que va en dirección noroeste hasta encontrar la orilla del océano.

La frontera terrestre parte, de acuerdo a lo estipulado en los instrumentos bilaterales precitados, de un punto en la orilla del Océano Pacífico. El punto 
inicial de la frontera terrestre entre ambos Estados se sitúa en la intersección de la orilla del Océano Pacífico (línea de la bajamar actual) con el arco de diez kilómetros de radio con centro en el puente sobre el río Lluta, que establece el límite terrestre en ese sector, como se grafica a continuación ${ }^{2}$.

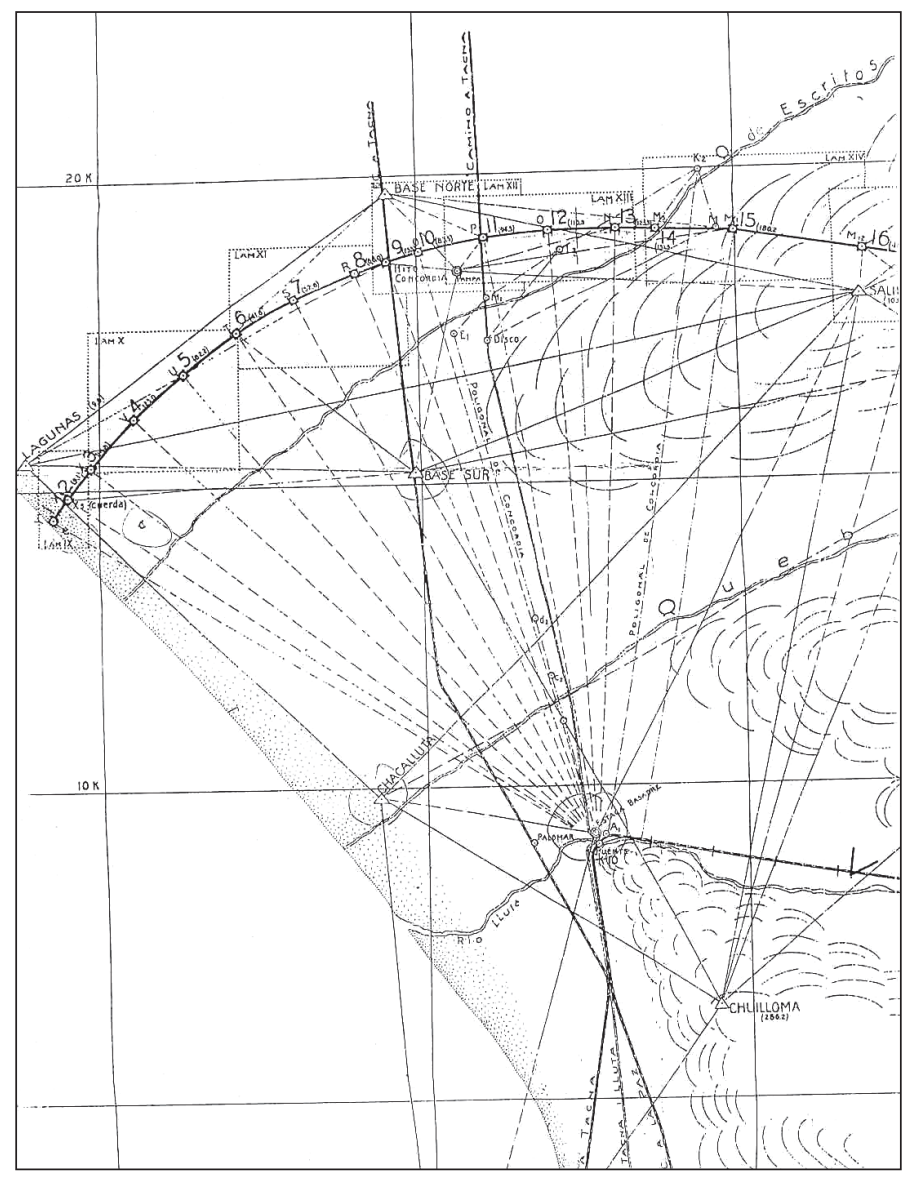

La diferencia de localización del punto de partida de la frontera terrestre con el primer hito colocado sobre dicha línea de frontera, obedece a que, siendo las orillas lugares sujetos a erosión y cambios de mareas, los hitos se colocan a cierta distancia de ellas, para preservarlos y darles un carácter permanente. Así, refiere Barberis:

2 BRIEBA, Enrique. Memoria sobre los límites entre Chile y Perú de acuerdo con el tratado del 3 de junio de 1929. Tomo III. Santiago de Chile: Instituto Geográfico Militar, 1931. 
Las señales o hitos que se colocan son, en general, pirámides de cemento o de hierro. Las comisiones demarcadoras no se valen solo de estas señales artificiales, sino que a veces recurren a accidentes geográficos. [...] En la erección de los hitos artificiales, las comisiones demarcadoras toman ciertas precauciones para evitar que estos sean desplazados o que desaparezcan y, si ello ocurre, para que puedan ser repuestos en el lugar adecuado ${ }^{3}$.

Por esta razón, el Hito $\mathrm{N}^{\circ} 1$ fue colocado por la Comisión Mixta de Límites entre el Perú y Chile a cierta distancia al noroeste del punto Concordia ubicado en la orilla del mar, en cumplimiento de las instrucciones recibidas por ambas Cancillerías, punto que es el inicio de la frontera terrestre, tal como se desprende de los instrumentos bilaterales analizados. Este uso, por lo demás, está plenamente reconocido por la práctica de los Estados, la jurisprudencia internacional y la doctrina de los publicistas.

\section{El cuestionamiento chileno al Tratado y Protocolo Complementario para resolver la cuestión de Tacna y Arica y su particular interpretación del inicio de la frontera terrestre desde 2001}

No obstante lo anteriormente señalado, Chile ha variado su posición de respeto al Tratado de 1929, poniendo en tela de juicio el punto de inicio de la frontera terrestre común, alegando que la misma parte del Hito $\mathrm{N}^{\circ} 1$, y no del punto Concordia en la intersección de la orilla del Océano Pacífico con el arco de 10 kilómetros hacia el noroeste del primer puente sobre el río Lluta de la vía férrea de Arica a La Paz, a diferencia de la posición jurídica del Perú, que invariablemente ha sostenido que el inicio de la frontera terrestre está en la orilla del Océano Pacífico.

En efecto, mediante notas diplomáticas enviadas a la Cancillería peruana en abril de 2001, como respuesta a la protesta peruana por la colocación de una caseta de vigilancia entre el Hito $\mathrm{N}^{\circ} 1$ y la orilla del mar, en territorio peruano, Chile cuestionó el punto de inicio de la frontera terrestre, desconociendo la literalidad del Tratado para resolver la cuestión de Tacna y Arica, que dispone que la frontera nace en la orilla del Océano

3 Barberis, Julio. El territorio del Estado y la soberanía territorial. Buenos Aires: Editorial Ábaco de Rodolfo Depalma, 2003, p. 173. 
Pacífico, y alegando que instrumentos posteriores, sin relación con el Tratado, habrían modificado dicho acuerdo ${ }^{4}$.

En consecuencia, el nuevo planteamiento chileno difiere sustancialmente con lo establecido por el Tratado para resolver la cuestión de Tacna y Arica de 1929 respecto del punto de inicio de la frontera terrestre, lo que está claramente graficado en el plano que firmó el Sr. Brieba, delegado chileno a la Comisión Mixta de Límites entre el Perú y Chiles, plano que se adjunta para mejor ilustración del lector.

Esta reciente posición chilena implica un claro desconocimiento de principios fundamentales consagrados en el derecho internacional. Así, en primer lugar tenemos el principio pacta sunt servanda, consagrado en el artículo 26 del Convenio de Viena de 1969 sobre derecho de los tratados, según el cual los tratados se celebran para ser cumplidos. Se trata de un principio fundamental reiteradamente afirmado en la práctica y en la jurisprudencia internacional, tanto arbitral como de la Corte Permanente de Justicia Internacional y de la Corte Internacional de Justicia; de una norma universalmente reconocida que constituye el fundamento moderno de la obligatoriedad de los tratados internacionales. Su importancia en el Derecho internacional es, en consecuencia, fundamental puesto que, cuando un tratado debidamente perfeccionado entra en vigor, se convierte en norma obligatoria para los Estados; ya no hay la menor posibilidad de desconocer sus efectos, porque con ello se pone en peligro la estabilidad del ordenamiento internacional, además de que el Estado incurriría en responsabilidad internacional.

Como seńala Scelle, el fundamento sociológico de la norma pacta sunt servanda radica en que proporciona seguridad jurídica a las relaciones internacionales y asegura relaciones pacíficas entre los pueblos; mientras su fundamento jurídico radica en la costumbre o en los principios generales del derecho ${ }^{6}$.

4 Rodríguez, Manuel. Delimitación marítima con equidad. El caso de Perú y Chile. Lima: Ediciones Peisa, 2007, pp. 207-208.

5 Véase nota 2.

6 SCelle, George. Manuel de Droit International Public. París: Domat - Montchrestien, 1948, p. 64; FIORE, Pasquale. Tratado de Derecho Internacional Público. Madrid: Centro Editorial de Góngora, Tomo I, 1894, p. 48; Mc NAIR, Arnold. The Law of Treaties. Londres, 1961, pp. 26-27. 


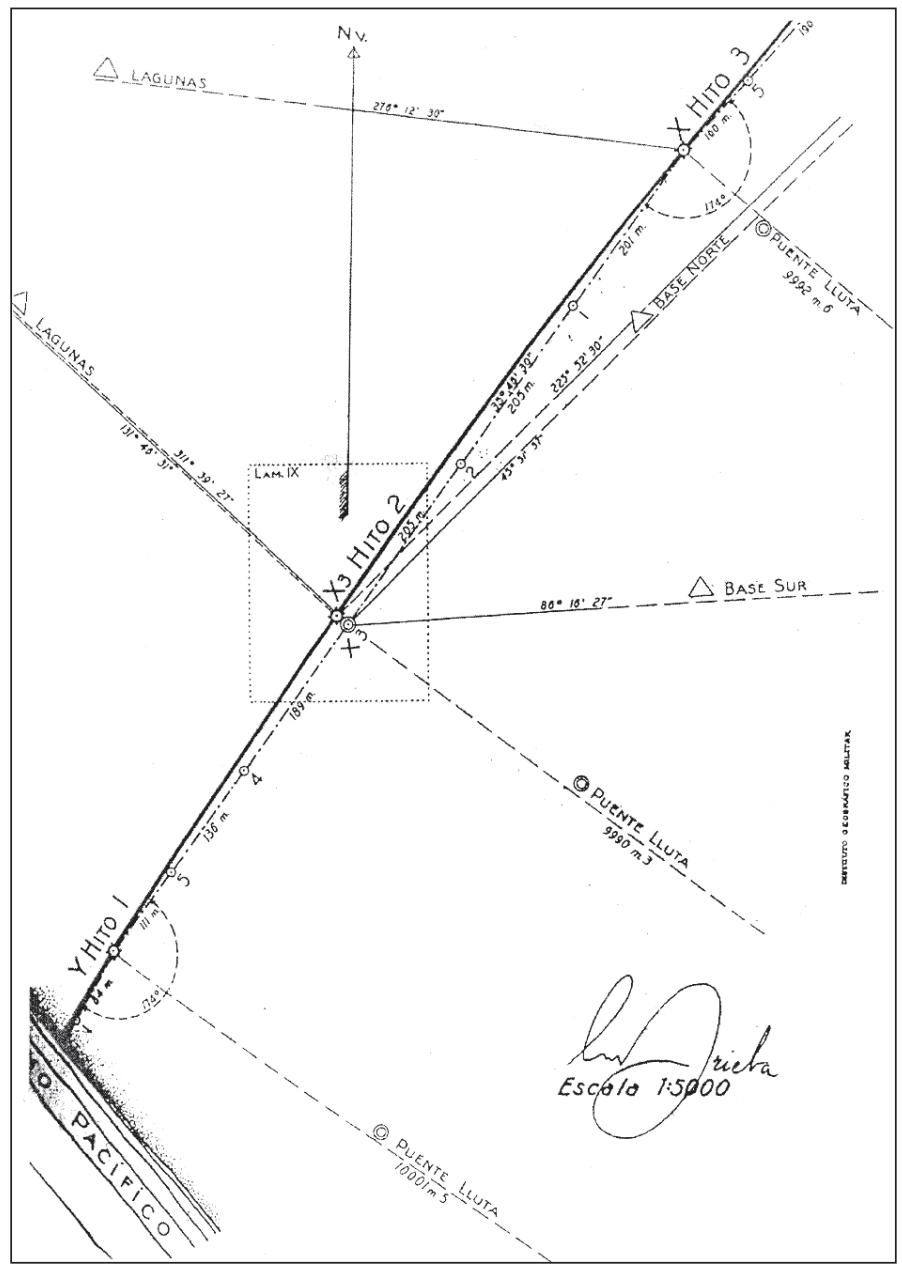

Plano firmado por el Sr. Enrique Brieba, delegado de Chile en la Comisión Mixta de Límites

Este principio cobra mayor importancia tratándose de acuerdos de límites $^{7}$. La jurisprudencia internacional ha confirmado la importancia jurídica de la estabilidad de los tratados de límites, como puede apreciarse de las sentencias de la Corte Internacional de Justicia en el Asunto del Templo de Preah Vihear: "De manera general, cuando dos países definen entre ellos

7 Novak, Fabián y Luis García Corrochano. Derecho Internacional Público. Tomo I: Introducción y Fuentes. Lima: Fondo Editorial de la PUC, 2000, p. 195. 
una frontera, uno de sus principales objetivos es alcanzar una solución estable y definitiva»"; en el Asunto de la Plataforma Continental del Mar Egeo: «Trátese de una frontera terrestre o del límite de la plataforma continental, la operación es esencialmente la misma; comporta el mismo elemento inherente de estabilidad y de permanencia y está sometida a la regla que un tratado de límites no es afectado por un cambio fundamental de las circunstancias"'; en el Asunto del diferendo territorial entre Libia y Chad donde el alto tribunal afirmó: «Una vez convenida, la frontera permanece, por lo que cualquier otro enfoque privará de efecto el principio fundamental de la estabilidad de las fronteras, cuya importancia la Corte ha subrayado en numerosas ocasiones ${ }^{10}$.

En segundo lugar, el ya citado artículo 26 de la Convención de Viena se refiere al principio de la buena fe, piedra angular del derecho, por el cual los tratados se interpretan y ejecutan de buena fe. La buena fe en las relaciones convencionales sugiere la actuación exenta de malicia, implica la observancia por las partes de determinado patrón de comportamiento razonable, sinceridad, honestidad, lealtad, en resumen, de ética a través de sus conductas. En consecuencia, este principio obliga a las partes en un tratado a abstenerse de realizar actos o plantear interpretaciones destinadas a incumplir o desnaturalizar los propósitos para los cuales este fue suscrito ${ }^{11}$.

En tercer lugar, este cuestionamiento pretende alterar unilateralmente un tratado de naturaleza perpetua como lo es el Tratado y Protocolo Complementario para resolver la cuestión de Tacna y Arica, firmado en Lima el 3 de junio de 1929, que establece el límite terrestre entre el Perú y Chile. Cabe resaltar que, de acuerdo a la costumbre internacional vigente desde aquella época, los tratados de límites y la demarcación que bajo su ministerio se efectúa no pueden ser modificados de manera unilateral, incluso, ni siquiera frente al hipotético caso de un cambio fundamental en las circunstancias, precepto que está recogido por el artículo 62.2.a) del

\footnotetext{
CIJ. Recueil, 1962, p. 34.

CIJ. Recueil, 1978, párrafo 85, pp. 36-37.

CIJ. Recueil, 1994, párrafo 72, p. 35.

1 Moncayo, Guillermo, Hortensia, GutiérRez Posse y Raúl Vinuesa. Derecho Internacional Público. Tomo I. Buenos Aires: Victor P. de Zavalía, 1981, p. 116; SChachter, Oscar. International Law in Theory and Practice. Dordrecht/Boston/London: Martinus Nijhoff, 1985, p. 130.
} 
Convenio de Viena de 1969, que establece: «Un cambio fundamental en las circunstancias no podrá alegarse como causa para dar por terminado un tratado o retirarse de él si el tratado establece una frontera». Al respecto, señala Reuter:

Apenas hay una razón teórica para que ciertos tratados escapen a priori a un posible desafío debido a un cambio en las circunstancias. Sin embargo, parece hacerse tradicionalmente la excepción en el caso de los tratados que versan sobre arreglos territoriales. [...] Si todo el derecho de los tratados depende de la interpretación de la voluntad presunta de las partes, entonces la excepción podrá aceptarse fácilmente: hay, en efecto, algunos tratados de cuyas partes puede suponerse razonablemente que han excluido ab initio todo desafío basado en el cambio en las circunstancias, y los tratados referentes a los convenios territoriales son un buen ejemplo de esto. El territorio define la esencia misma del Estado; por tanto, puede presumirse que las partes procuran descartar todo cambio posterior en las circunstancias ${ }^{12}$.

Como se sabe, los tratados que establecen una frontera y los tratados de paz son considerados por la doctrina y la jurisprudencia internacional como acuerdos de naturaleza perpetua, que no pueden ser modificados unilateralmente, ni los Estados partes pueden retirarse de ellos por medio de la denuncia ${ }^{13}$. Y es que el Derecho Internacional busca dar seguridad jurídica a las relaciones entre Estados, máxime cuando se trata de asuntos especialmente sensibles de las relaciones internacionales.

No obstante todo lo expuesto, Chile ha decidido poner en cuestión todos estos principios rectores del derecho internacional.

12 Reuter, Paul. Introducción al derecho de los tratados. México D.F.: FCE, 1999, p. 219.

13 Arellano García, Carlos. Primer Curso de Derecho Internacional Público. México: Porrúa, 1993, p. 640; Comisión de Derecho Internacional. Anuario, tomo 2, 1963, p. 64. BARBERIS, Julio. El territorio del Estado y la soberanía territorial. Buenos Aires: Editorial Ábaco de Rodolfo Depalma, 2003, p. 166: El derecho de los tratados contiene dos disposiciones que rigen particularmente para los convenios sobre límites internacionales, a saber: la no aplicabilidad de la cláusula rebus sic stantibus y la imposibilidad de su denuncia unilateral. Quoc Dinh, Nguyen, Patrick Dailler y Alain Pellet. Droit international public. París: LGDJ, 1994, p. 298: Existen tratados que, en razón de su naturaleza, no son susceptibles de denuncia, como los tratados de paz o aquellos que fijan fronteras. VIRALLY, Michel. «Panorama du droit international contemporain. Cours General de droit international public». RCADI, 1983 - V, Tomo 183, p. 150. En todos los casos, los tratados relativos a fronteras han visto reconocida una naturaleza jurídica particular por el derecho internacional, que les atribuye una estabilidad mayor que aquella de los tratados ordinarios. 
Por otro lado, la posición chilena también desconoce otro principio jurídico, heredado de la tradición romana, según el cual las cosas se deshacen de la misma forma en que se hicieron. $\mathrm{Al}$ respecto, cabe resaltar que dicho tratado, al ser de límites, es de naturaleza solemne, por lo que fue aprobado por los poderes legislativos de ambos Estados partes, antes de ser ratificado por los jefes de Estado de los dos países ${ }^{14}$.

Si bien esta nueva posición chilena en torno al inicio de la frontera terrestre resulta jurídicamente insostenible, solo se explica por el interés de dicho país de ocultar una serie de incongruencias en su planteamiento del paralelo geográfico como supuesto límite marítimo entre el Perú y Chile, como tendremos ocasión de analizar en el siguiente punto.

\section{Consecuencias de la pretensión chilena respecto del límite terrestre y marítimo. La formación de una "costa seca»}

Como se sabe, la pretensión chilena del paralelo geográfico como supuesto límite marítimo entre ambos Estados genera el absurdo jurídico y geográfico de recortar las 200 millas de dominio marítimo del Perú en forma progresiva, a medida que se recorre la costa peruana de norte a sur.

Sin embargo, este no es el único absurdo que se deriva de la posición chilena. En efecto, la variación de la posición chilena en torno al límite terrestre se explica por el interés en darle consistencia a su planteamiento de una frontera marítima coincidente con el paralelo geográfico. En efecto, cuando Chile sostiene que la frontera terrestre se inicia en el Hito $\mathrm{N}^{\circ}$ 1 y luego coloca una caseta de vigilancia en territorio peruano (que retiró de inmediato ante la protesta del gobierno peruano), entre el Hito $\mathrm{N}^{\circ} 1$ y el inicio de la frontera en la orilla del mar, lo que persigue es presentar como un hecho consumado que, desde el Hito $\mathrm{N}^{\circ} 1$ se trace un paralelo hacia la orilla del mar, significando una nueva línea de frontera terrestre, y que a partir de ese punto en la orilla del mar, se trace un paralelo como frontera marítima seudo convencional entre ambos Estados; de tal forma,

14 Tanto el derecho interno de los Estados involucrados, como el Derecho Internacional prevén una fórmula solemne para la celebración de tratados que aborden temáticas complejas, como son los asuntos de límites. De LA Guardia, Ernesto. Derecho de los Tratados Internacionales. Buenos Aires: Editorial Ábaco de Rodolfo de Palma, 1997, p. 161. 
el Hito $\mathrm{N}^{\circ} 1$ serviría como referencia limítrofe de la frontera terrestre y marítima.

La pretensión chilena daría lugar a la formación de un triángulo terrestre de casi dos hectáreas cuyos vértices serían: el Hito $\mathrm{N}^{\circ} 1$, ubicado tierra adentro, el punto "Concordia», situado en la Orilla del Océano Pacífico, y un punto en la orilla del mar paralelo al Hito $\mathrm{N}^{\circ} 1$, territorio que pretenderían de dominio chileno, enajenando ese espacio al Perú y adulterando la frontera reconocida desde 1930, que parte del punto «Concordia» y describe un arco hacia el Hito $\mathrm{N}^{\circ} 1$, constituyendo el límite internacionalmente reconocido entre ambos Estados.

La intención de toda esta tour de force sería ocultar una realidad, cual es que la tesis chilena del paralelo, aunada a su nueva tesis del Hito $\mathrm{N}^{\circ} 1$ como punto de inicio de la frontera terrestre, forman absurdamente un triangulo terrestre peruano cuya costa sería bańada por mar chileno. En otras palabras, el Perú tendría una "costa sin mar» o "costa seca» o, si se quiere, Chile tendría un «mar sin costa». Esta pretensión, además de ser jurídicamente insostenible, es geográficamente imposible pues plantea un absurdo, ya que divorcia la geografía al separar la costa del mar que lo baña.

En efecto, tanto la doctrina como la jurisprudencia internacional han sido muy claras en rechazar cualquier planteamiento destinado a la formación de una «costa seca». Así tenemos, el Asunto del Canal del Beagle (1977), entre Argentina y Chile donde, coincidentemente, se pretendió sin éxito sustentar esta tesis ${ }^{15}$. El tribunal arbitral, como era de esperarse, rechazó esta pretensión, siguiendo el principio jurídico que la tierra domina sobre el mar.

Chile, entonces, habría aprendido la lección, de ahí su interés por afirmar, con la colocación de una caseta, que el referido triángulo es chileno y no peruano.

15 «Dispute between Argentina and Chile concerning the Beagle Channel». Reports of International Arbitral Awards. 18 February 1977. Volume XI, § 107, pp. 144-145. 


\section{El cumplimiento del Tratado para resolver la cuestión de Tacna y Arica y su incidencia en la determinación de la frontera marítima}

Como ha quedado demostrado en el punto anterior, la tesis chilena sobre el punto de inicio de la frontera terrestre no solo transgrede el Tratado para resolver la cuestión de Tacna y Arica, firmado en Lima el 3 de junio de 1929 y la demarcación establecida de común acuerdo entre ambos Estados, sino también principios y normas consagrados por el derecho internacional público. En consecuencia, debemos descartar esta postura para fundamentar el inicio de la frontera marítima entre ambos Estados.

Como han establecido la jurisprudencia internacional y la doctrina, para la determinación de una frontera marítima es necesario que previamente exista acuerdo sobre el punto terrestre desde el cual ha de partir dicha frontera. Siguiendo el principio que la tierra domina sobre el mar, para la determinación de una frontera marítima — proyección del dominio terrestre- es necesaria la delimitación previa del territorio estatal, y numerosos contenciosos internacionales de carácter territorial y marítimo testimonian dicha prelación al resolver primero los temas territoriales y luego, y en función de ellos, los temas marítimos.

En consecuencia, en el diferendo sobre delimitación marítima que sostienen Perú y Chile se deberá partir del punto terrestre establecido por el Tratado para resolver la cuestión de Tacna y Arica, firmado en Lima el 3 de junio de 1929, desde el cual debe partir nuestra frontera marítima. Por las razones expuestas, este punto no puede ser el que actualmente sostiene Chile. Por el contrario, es el planteamiento del Perú, ajustado a dicho instrumento bilateral y al derecho internacional público, el único que puede aplicarse.

En este sentido, siguiendo lo establecido en el Tratado para resolver la cuestión de Tacna y Arica, de las instrucciones emitidas por los ministerios de Relaciones Exteriores de ambos Estados y el Acta Final de la Comisión Mixta de Límites entre el Perú y Chile, la frontera terrestre nace en el punto "Concordia», y es a partir de este punto que la Corte Internacional de Justicia deberá determinar el sentido y proyección de la frontera marítima entre ambos Estados, de acuerdo a los criterios internacionalmente aceptados para solucionar dichos contenciosos. 